\title{
56. The Study of Cerebral Venous Disorder. Intracranial Abnormal Venous Network
}

\author{
Cerebral Venous Dysgenesis \\ Mizuo Kagawa. Toyoaki Shinohara, Nobuko Ogawa, \\ Toshio Beppu, and Koichi Kitamura \\ Department of Neurosurgery, Tokyo Women's \\ Medical College, Tokyo
}

In this paper, we intended to clarify a cerebral vascular disorder due to pathological venous vasculature. 18 cases with abnormal cortical venous network was presented and the pathogenesis of this disease was discussed.

Clinical manifestations of this disease are mental retardation, rigospasticity, convulsive disorder and amaurosis. The disease occurrs in young decade, espicially in children under 8 years of age. However no sex predominance is noted. Angiograpic findings of this disease are extremely characteristic, they are, early venous filling with fine capillary stain in subcortical region, marked venous abnormal network akin to embryonic venous network of the cortex and nonvisualization of deep veins, especially basal vein. This cortical venous abnormal network consists of fine spiral, tortuous venule, which drains into abnormal anastomotic vein or brindging vein of the cortex. Numerous pathological anstomosis between those abnormal vein and venules is elicited. Venous sinus occlusion has not been shown, as far as morphological study is concerned, these abnormal cortical venous network is quite different from collateral circulation in venous sinus occlusion or venous occlusive disease.

We call this pathological venous network as "cerebral venous dysgenesis" tentatively. The following pathogenesis should be considered.

1) Capillary angiomatous malformation, persistent embryonic venous network, due to developmental disturbance of the brain.

2) Agenesis or occlusive disease of deep crebral vein.

3) Reactive proliferation of venous channels due to subdural effusion, or infection at perinatal period.

The pathogenesis of this disease has not been clarified yet, and further angiographic and clinico-pathological survey should be required.

\section{Total Removal of the Gerebral Arteriovenous Malformation}

\author{
Jiro Suzuki, Takashi Iwabuchi, Akira Takaku, \\ Sigeaki Hori, So Sato and Namio Kodama \\ Institute of Brain Diseases, Tohoku University \\ School of Medicine


In the treatment of $\mathrm{A}-\mathrm{V}$ malformation, it is accepted for total extirpation to be effective.

But if $\mathrm{A}-\mathrm{V}$ malformation is located in the deep portion or in an important area of cerebrum, only conservative therapy, such as clipping of afferent artery or injection of plastic material, is performed.

It is true that there are some cases in which it is impossible to carry out total extirpation, but it is a duty for neurosurgeons to expand the operative indication by some method. In this film ( $16 \mathrm{~mm}$ techni-color, optical sound, $19 \mathrm{~min}$.) four cases in which we have carried out total extirpation by a new method will be shown.

Under $27^{\circ} \mathrm{C}$ hypothermia, craniotomy is carried out. Afferent main artery from Willis' ring at the base of brain is exposed and temporary clipping is done.

Then total extirpation of A-V malformation is performed easily in dry field, with brain tissue uninjured as much as possible. In the case of $\mathrm{A}-\mathrm{V}$ malformation, the permitted time of temporary clipping of cerebral blood flow is rather long.

In one case the clipping time was 95 minutes under $27^{\circ} \mathrm{C}$ hypothermia, but no postoperative disturbance was found. By this method the operation is performed in such $\mathrm{A}-\mathrm{V}$ malformation of considerable risk as in deep portion of Sylvian fissure, in motor area, in motor area facing the falx, in deep portion of temporal lobe or accompanied with cerebral aneurysm.

Total extirpation of A-V malformation were succesfully performed in all these cases with no postoperative disturbance at all. In this film total extirpation of A-V malformation in deep portion or in an important area of cerebrum is shown, which has been thought to be diffecult to carry out.

As for the A-V malformation located in midline deep portion of cerebrum drainning into deep cerebral vein and/or diffuse A-V malformation, the succesful treatment must depend upon future therapeutical development.

\title{
58. Surgical Treatment of Intracranial Arteriovenous Malformation
}

\author{
Norihiko Basugr, Isamu Sarto, and Keiji Sano \\ Department of Neurosurgery, University of Tolyo
}

Since 1949 to 1971 (during 22 years), 177 cases of intracranial arteriovenous malformation (AVM) were treated in our clinic. 143 out of these 177 cases $(81 \%$ ) underwent surgical treatment as follows; extirpation-59 cases and other palliative operations such as ligation of afferent and efferent vessels (53 cases), artificial embolization (17 cases), cervical carotid ligation and decompression (7 cases). Operative mortality was $4 \%(6 / 143)$ in these surgical treatment.

In this report follw-up results were described. Surgical indication and treatment of AVM were discussed. 